\title{
High-throughput screening of monoclonal antibodies against plant cell wall glycans by hierarchical clustering of their carbohydrate microarray binding profiles
}

\author{
Isabel Moller • Susan E. Marcus • Ash Haeger • \\ Yves Verhertbruggen • Rene Verhoef • Henk Schols • \\ Peter Ulvskov • Jørn Dalgaard Mikkelsen • \\ J. Paul Knox • William Willats
}

Received: 27 March 2007 /Revised: 7 June 2007 / Accepted: 19 June 2007 / Published online: 14 July 2007

(C) Springer Science + Business Media, LLC 2007

\begin{abstract}
Antibody-producing hybridoma cell lines were created following immunisation with a crude extract of cell wall polymers from the plant Arabidopsis thaliana. In order to rapidly screen the specificities of individual monoclonal antibodies (mAbs), their binding to microarrays containing 50 cell wall glycans immobilized on nitrocellulose was assessed. Hierarchical clustering of microarray binding profiles from newly produced mAbs, together with the profiles for $\mathrm{mAbs}$ with previously defined specificities allowed the rapid assignments of $\mathrm{mAb}$ binding to antigen classes. mAb specificities were further investigated using
\end{abstract}

I. Moller $\cdot$ W. Willats $(\bowtie)$

The Department of Molecular Biology,

The University of Copenhagen,

Ole Maaløes Vej 5,

2200 Copenhagen, Denmark

e-mail: willats@my.molbio.ku.dk

S. E. Marcus - A. Haeger - Y. Verhertbruggen - J. P. Knox

Centre for Plant Sciences, University of Leeds,

Leeds LS2 9JT, UK

P. Ulvskov

The Biotechnology Group, University of Aarhus,

Thorvaldsensvej 40,

1871 Frederiksberg, Denmark

R. Verhoef $\cdot H$. Schols

Department of Agrotechnology and Food Sciences, Laboratory of Food Chemistry, Wageningen University, 6700 EV Wageningen, The Netherlands

J. D. Mikkelsen

Danisco A/S,

Langebrogade 1,

Copenhagen, Denmark subsequent immunochemical and biochemical analyses and two novel mAbs are described in detail. mAb LM13 binds to an arabinanase-sensitive pectic epitope and mAb LM14, binds to an epitope occurring on arabinogalactan-proteins. Both mAbs display novel patterns of recognition of cell walls in plant materials.

Keywords Carbohydrate microarrays · Plant cell walls . Monoclonal antibodies $\cdot$ Hierarchical clustering

\section{Introduction}

Carbohydrate microarrays provide a means of rapidly screening the interactions between glycans and other molecules [17]. Applications for this technology include the screening of protein-glycan interactions, characterization of carbohydrateactive enzymes and the analysis of the specificities of monoclonal antibodies (mAbs) and carbohydrate-binding modules $[8,9]$. mAbs are powerful tools for investigating the biological roles of glycans but producing them is time consuming, labour-intensive and costly. Hybridoma-based $\mathrm{mAb}$ production involves the fusion of spleen cells from an immunized animal with myeloma cells. The resulting hybridoma cell lines are both immortal and secrete antibody into the cell supernatant [10]. This procedure is conventionally performed in a one-by-one fashion, such that each animal is immunized with a single antigen and the resulting antibodies are screened for desired specificities using enzyme-linked immunosorbent assays (ELISAs). However ELISA-based screening is low throughput because only a limited amount $(\sim 100 \mu \mathrm{l})$ of hybridoma supernatant is available for screening during the initial stages of $\mathrm{mAb}$ 
production and this is typically only sufficient to test each $\mathrm{mAb}$ against just one or two antigens. An alternative approach involves 'shotgun' immunisation with a mixture of antigens [11]. This potentially results in the simultaneous generation of antibodies with a range of specificities, but the time limiting step then becomes the detailed retrospective screening of their specificities. However, microarrays offer a means of rapidly screening limited amounts of hybridoma supernatant against multiple antigens and therefore greatly increasing throughput in the identification of valuable cell lines. Here we report the use of shotgun immunisation followed by microarray-based screening of hybridoma supernatants in order to produce mAbs against plant cell wall glycans.

Plant cell walls are fibre composites that contain some of the most complex glycans known [12, 13]. In addition to their biological roles, many cell wall components have important industrial applications including as functional food ingredients, pharmaceuticals, nutriceuticals, fibres and increasingly, bio-fuels [14-16]. Cell wall glycans can be broadly grouped into cellulose, hemicelluloses, pectins and glycoproteins [17]. Cellulose microfibrils are cross-linked by hemicelluloses such as xyloglucans, xylans and mixed linkage glucans forming a tough load-bearing matrix which is embedded in pectic polysaccharides [18]. Pectins are the most complex and heterogeneous family of cell wall glycans and are comprised of a series of galacturonic acid-rich polymers including homogalacturonan (HG), rhamnogalacturonan I (RGI), rhamnogalacturonan-II (RGII) and xylogalacturonans (XG). In addition, structurally complex arabinan, galactan and arabinogalactan polymers may be present as side chains to the galacturonan-rich backbone domains [18]. The protein moieties of cell wall glycoproteins are often rich in hydroxyproline (Hyp) and these polymers, which are collectively referred to as Hyp-rich glycoproteins (HRGPs), include the extensins and arabinogalactanproteins (AGPs) [19-21].

The fine structures and relative amounts of cell wall components vary greatly not only among plants, but also between organs, tissues, cells, and even between different micro-domains within a single cell wall. This complexity and heterogeneity presents a major barrier to detailed analysis and our understanding of many aspects of plant cell wall structure and function is far from complete. Several mAbs with specificities for diverse plant cell wall components have been developed and these are powerful tools for the analysis of cell walls [22]. However, the repertoire of mAbs currently available covers only a small proportion of the glycan structures that have been identified and there is a pressing need for a wider range of $\mathrm{mAbs}$ to facilitate the further characterization of cell walls. We have developed a method of rapidly screening $\mathrm{mAb}$ specificities using microarrays of cell wall glycans including pectins with different degrees and patterns of methyl-esterification, pectic side chains (e.g. arabinan and galactan), hemicelluloses (e.g. xylans, mannans, and xyloglucans), HRGPs (e.g. AGP-rich gums), $\beta$-linked glucans $($ e.g. $(1 \rightarrow 3)(1 \rightarrow 4)$ - $\beta$-glucan and $(1 \rightarrow 3)$ - $\beta$-glucan) and celluloses (e.g. hydroxylethyl cellulose and carboxylmethyl cellulose). A further 14 samples were sequentially extracted from $A$. thaliana using CDTA and $\mathrm{NaOH}$ which are known to predominately solublize pectic polymers and hemicelluloses respectively [23].

\section{Experimental procedures}

Immunisation and generation of monoclonal hybridomas

Cell wall polymers were isolated from 6 week old wild type A. thaliana plants, ecotype Col-0, grown in soil at $22^{\circ} \mathrm{C}$ with cycles of $10 \mathrm{~h}$ of light/14 h of darkness. One $\mathrm{g}$ dry weight of a mixture of leaves, stems and roots was homogenized to a fine powder in liquid nitrogen. The homogenate was incubated with $20 \mathrm{ml} 50 \mathrm{mM} \mathrm{1,2-}$ Diaminocyclohexanetetraacetic acid (CDTA; pH 7.5) for $3 \mathrm{~h}$ at $18^{\circ} \mathrm{C}$ and centrifuged for $20 \mathrm{~min}$ at $4,400 \mathrm{rpm}$. The supernatant was collected and dialyzed extensively against deionized water $\left(\mathrm{dH}_{2} \mathrm{O}\right)$ in dialysis tubing $(6-8,000 \mathrm{kDa}$ molecular weight cut off) to remove low molecular weight molecules and freeze dried. The material was dissolved in phosphate-buffered saline (PBS) to generate the immunogen. Rats were used for antibody production so as to make subsequent comparisons with existing mAbs, most of which were produced in rats, as valid as possible. The immunization of rats, hybridoma preparation and cloning procedures were as described previously [24]. Briefly, two male Wistar rats were each injected subcutaneously with $250 \mu \mathrm{l}$ of an emulsion of the isolated cell wall material at $1 \mathrm{mg} / \mathrm{ml}$ in PBS with an equal volume Freund's complete adjuvant on day 0 . On days 40 and 79 the injections were repeated using incomplete adjuvant. Tail bleeds were taken 10 days after injections to assess the immune response. On day 198 a pre-fusion boost was given to the selected rat and 3 days later, the spleen was removed and lymphocytes were isolated and fused with rat myeloma cell line IR983F [25] using standard polyethylene glycol fusion of lymphocytes and myeloma cells. Hybridoma lines were initially screened by ELISA with the immunogen coated onto microtitre plates (MaxiSorp, Nunc, Roskilde, Denmark) at $50 \mu \mathrm{g} / \mathrm{ml}$.

Previously described monoclonal antibodies

Arrays were probed with $23 \mathrm{mAbs}$ with previously described specificities and details of these are provided in Table 1. All were rat antibodies except mAbs CCRC-M1, BS-400-02, BS-400-03, and BS-400-04 which were produced in mice and PAM1 which was produced by phage display. 
Table 1 Previously characterized monoclonal antibodies used to probe glycan arrays. $\mathrm{HG}$, homogalacturonan

\begin{tabular}{lll}
\hline Specificity & Name & Ref. \\
\hline Un-esterified HG & PAM1 & {$[31]$} \\
Un-esterified/Calcium ion cross-linked HG & 2 F4 & {$[34]$} \\
Partially methyl-esterified HG & JIM5 & {$[33]$} \\
Partially methyl-esterified HG & JIM7 & {$[33]$} \\
$(1 \rightarrow 4)-\beta$-galactan & LM5 & {$[37]$} \\
$(1 \rightarrow 5)-\alpha$-arabinan & LM6 & {$[24]$} \\
Fucosylated xyloglucan & CCRC-M1 & {$[38]$} \\
Non-fucosylated xyloglucan & LM15 & \\
$(1 \rightarrow 4)-\beta$-mannan/galacto- $(1 \rightarrow 4)-\beta-$ mannan & BS-400-4 & {$[39]$} \\
$(1 \rightarrow 4)-\beta$-xylan & LM10 & {$[40]$} \\
$(1 \rightarrow 3)(1 \rightarrow 4)-\beta$-glucan & BS 400-3 & {$[41]$} \\
$(1 \rightarrow 3)-\beta$-glucan & BS 400-2 & {$[32]$} \\
$(1 \rightarrow 4)-\beta$-xylan/arabinoxylan & LM11 & {$[40]$} \\
Arabinogalactan-protein & JIM4 & {$[43]$} \\
Arabinogalactan-protein & LM2 & {$[42]$} \\
Arabinogalactan-protein & MAC207 & {$[43]$} \\
Arabinogalactan-protein & JIM8 & {$[44]$} \\
Arabinogalactan-protein & JIM13 & {$[43]$} \\
Arabinogalactan-protein & JIM16 & {$[43]$} \\
Arabinogalactan-protein & JIM14 & {$[43]$} \\
Extensin & LM1 & {$[45]$} \\
Extensin & JIM19 & {$[46]$} \\
Extensin & JIM20 & {$[46]$} \\
\hline & &
\end{tabular}

Glycan samples used on the array

Details of the 50 defined glycans used are provided in Table 2. Most glycans were dissolved in $\mathrm{dH}_{2} \mathrm{O}$. Arabinoxylan and glucuronoxylan were prepared by boiling in $\mathrm{dH}_{2} \mathrm{O}$ for $10 \mathrm{~min}$. and then standing for $3 \mathrm{~h}$ at $18^{\circ} \mathrm{C}$ before use. Glucomannan was prepared by wetting with $95 \%$ ethanol followed by addition of $\mathrm{dH}_{2} \mathrm{O}$. The mixture was heated to boiling point and stirred for $20 \mathrm{~min}$ until dissolved. Pachyman was prepared by dissolution in a minimal volume of $10 \%(w / v)$ sodium hydroxide followed by neutralization with acetic acid. 14 samples on the arrays were cell wall polymers extracted from A. thaliana organs listed in Table 2 using CDTA and $4 \mathrm{M} \mathrm{NaOH}$. Fifty milligrams (fresh weight) of each organ collected from at least four separate plants were homogenized to a fine powder prior to adding $300 \mu \mathrm{l}$ of $50 \mathrm{mM}$ CDTA (pH 7.5). After incubating with rotation for $4 \mathrm{~h}$ at $20^{\circ} \mathrm{C}$, the extracts were centrifuged at $4,400 \mathrm{rpm}$ for $10 \mathrm{~min}$ and the supernatants ('CDTA extracts') removed. Pellets were resuspended in $300 \mu \mathrm{l}$ of $4 \mathrm{M} \mathrm{NaOH}$ and samples were incubated with rotation for $4 \mathrm{~h}$ at $20^{\circ} \mathrm{C}$ prior to centrifugation at 4,400 rpm for $10 \mathrm{~min}$. Supernatants were ' $\mathrm{NaOH}$ extracts'.

Post-printing modification of glycans

Glycan samples on selected arrays were modified in situ after printing by enzymatic digestion. For the data in Fig. 6 selected arrays were digested with endo- $\alpha(1-5)$-L-arabinanase or endo- $\beta(1-4-)$-galactanase (both from Aspergillus niger, Megazyme (Bray, Ireland) used at $1 \mathrm{U} / \mathrm{ml}$ in $200 \mathrm{mM}$ sodium acetate $\mathrm{pH}$ 4.0.

\section{Printing of arrays}

Glycans were applied to nitrocellulose membrane $(0.45 \mu \mathrm{m}$ pore size, Schleicher and Schuell, Dassel, Germany) at two concentrations $(0.2$ and $0.04 \mathrm{mg} / \mathrm{ml})$ and in duplicate such that each sample was represented by four spots. CDTA and $\mathrm{NaOH}$ extracted $A$. thaliana material was printed as extracted and as a five fold dilution, also in duplicate. Printing was performed using a microarray robot (Microgrid II, Genomic Solutions, Ann Arbor, MI, USA) equipped with split pins (MicroSpot 2500 , Genomic Solutions). Pins were washed twice in $\mathrm{dH}_{2} \mathrm{O}$ after deposition of each sample.

\section{Probing of arrays}

Arrays were blocked by incubation for $1 \mathrm{~h}$ in PBS $(140 \mathrm{mM}$ $\mathrm{NaCl}, 2.7 \mathrm{mM} \mathrm{KCl}, 10 \mathrm{mM} \mathrm{Na} 2 \mathrm{HPO}_{4}, 1.7 \mathrm{mM} \mathrm{KH}_{2} \mathrm{PO}_{4}$, $\mathrm{pH} 7.5)$ containing $5 \% \mathrm{w} / \mathrm{v}$ low fat milk powder $(5 \%$ MPBS). Arrays were then probed for $2 \mathrm{~h}$ with antibodies diluted in 5\%MPBS. All antibodies were used as $1 / 10$ dilutions except CCRC-M1 which was used at $1 / 50$, and BS-400-2, BS-400-3 and BS-400-4 which were used at 1/ 200. After washing with PBS, arrays were incubated for $2 \mathrm{~h}$ in either anti-rat or anti-mouse secondary antibody conjugated to alkaline phosphatase (Sigma, Poole, UK) diluted 1/ 5000 in 5\%MPBS. After washing in PBS, arrays were developed using a substrate containing 5-bromo,4-chloro,3indolylphosphate (BCIP) and nitroblue tetrazolium (NBT) in BCIP/NBT buffer $\left(100 \mathrm{mM} \mathrm{NaCl}, 5 \mathrm{mM} \mathrm{MgCl}_{2}, 100 \mathrm{mM}\right.$ diethanolamine, $\mathrm{pH}$ 9.5).

Scanning and analysis

Arrays were scanned, converted to 16 bit grey-scale TIFFs, transformed to negative images and uploaded into ImaGene 6.0 microarray analysis software (BioDiscovery, El Segundo, CA, USA). Semi-automatic gridding was used to create an analysis area for each spot, and a 5-pixel zone around each spot was used for calculation of local background signals. Individual spot signals were defined as the mean pixel value within each spot area (red zone in Fig. 1d) minus the median pixel value in the surrounding local background area (green zone in Fig. 1d). The mean of the four individual spot signals for each sample was defined as the 'mean sample value' (MSV) and 'total mean sample values' (TMSVs) were the means of MSVs from three separate experiments. Selected data sets (TMSVs) were used to generate heatmaps (Figs. $2 \mathrm{~b}$ and $5 \mathrm{~b}$ ) using online heat- 
Table 2 Samples included on the glycan arrays

\begin{tabular}{|c|c|}
\hline Alphanumerical codes & Samples \\
\hline A1 & Arabinan (sugar beet) \\
\hline B1 & Pectin (apple) \\
\hline C1 & Galactan (lupin) \\
\hline D1 & Homogalacturonan (sugar beet) \\
\hline E1 & Pectin (lime) B15 \\
\hline F1 & Pectin (lime) B43 \\
\hline G1 & Pectin (lime) B71 \\
\hline H1 & Pectin (lime) 96 \\
\hline A2 & Pectin (lime) F11 \\
\hline B2 & Pectin (lime) F19 \\
\hline $\mathrm{C} 2$ & Pectin (lime) F43 \\
\hline D2 & Pectin (lime) F76 \\
\hline E2 & Pectin (lime) P16 \\
\hline F2 & Pectin (lime) P24 \\
\hline G2 & Pectin (lime) P32 \\
\hline $\mathrm{H} 2$ & Pectin (lime) P41 \\
\hline $\mathbf{A 3}$ & Pectin (lime) P46 \\
\hline B3 & Pectin (lime) P60 \\
\hline C3 & Pectin (lime) P76 \\
\hline D3 & RGI (soybean) \\
\hline E3 & RGII (A. thaliana) \\
\hline F3 & Xylogalacturonan (pea) \\
\hline G3 & MHR I (apple) \\
\hline H3 & MHR II (carrot) \\
\hline A4 & MHR III (potato) \\
\hline B4 & MHR HS1 (apple) \\
\hline $\mathrm{C} 4$ & MHR HS2 (apple) \\
\hline D4 & Xylogalacturonan (apple) \\
\hline E4 & AGP (P. patens) \\
\hline F4 & Seed mucilage $(A$. thaliana $)$ \\
\hline G4 & Xyloglucan/mannan (tomato) \\
\hline H4 & Glucomannan (konjac) \\
\hline A5 & Gum (guar) \\
\hline B5 & Gum (locust bean) \\
\hline C5 & Gum arabic (acacia) \\
\hline D5 & Gum (karaya) \\
\hline E5 & Gum (tragacanth) \\
\hline F5 & AGP (larch) \\
\hline G5 & Arabinoxylan (wheat) \\
\hline H5 & $\beta(1-3),(1-4)$-glucan (lichenan) \\
\hline A6 & Mannan (ivory nut) \\
\hline B6 & Xyloglucan (tamarind) \\
\hline C6 & Glucuronoarabinoxylan (maize) \\
\hline D6 & Hydroxyethyl cellulose \\
\hline E6 & $\beta(1-4)$-glucan (avicel) \\
\hline F6 & Carboxymethyl cellulose \\
\hline G6 & Alginic acid \\
\hline H6 & $\beta(1-3),(1-6)$-glucan (laminarin) \\
\hline A7 & $\beta(1-3)$-glucan (pachyman) \\
\hline B7 & $\beta(1-4),(1-6)$-glucan (pullulan) \\
\hline C7 & CDTA extract (A. thaliana flowers) \\
\hline D7 & CDTA extract (A. thaliana siliques) \\
\hline E7 & CDTA extract (A. thaliana stem top) \\
\hline F7 & CDTA extract (A. thaliana stem middle) \\
\hline G7 & CDTA extract ( $A$. thaliana stem base) \\
\hline H7 & CDTA extract (A. thaliana leaves) \\
\hline A8 & CDTA extract (A. thaliana roots) \\
\hline B8 & $\mathrm{NaOH}$ extract (A. thaliana flowers) \\
\hline C8 & $\mathrm{NaOH}$ extract (A. thaliana siliques) \\
\hline D8 & $\mathrm{NaOH}$ extract $(A$. thaliana stem top) \\
\hline E8 & $\mathrm{NaOH}$ extract (A. thaliana stem middle) \\
\hline F8 & $\mathrm{NaOH}$ extract (A. thaliana stem base) \\
\hline G8 & $\mathrm{NaOH}$ extract $(A$. thaliana leaves $)$ \\
\hline H8 & $\mathrm{NaOH}$ extract $(A$. thaliana roots) \\
\hline
\end{tabular}

Alphanumerical codes refer to the position of samples on arrays. Source organisms are in parentheses

$R G I$ Rhamnogalcturonan I; RGII rhamnogalacturonan II; $M H R$ modified hairy region; $A G P$ arabinogalactan-protein a $22 \mathrm{~mm}$

b
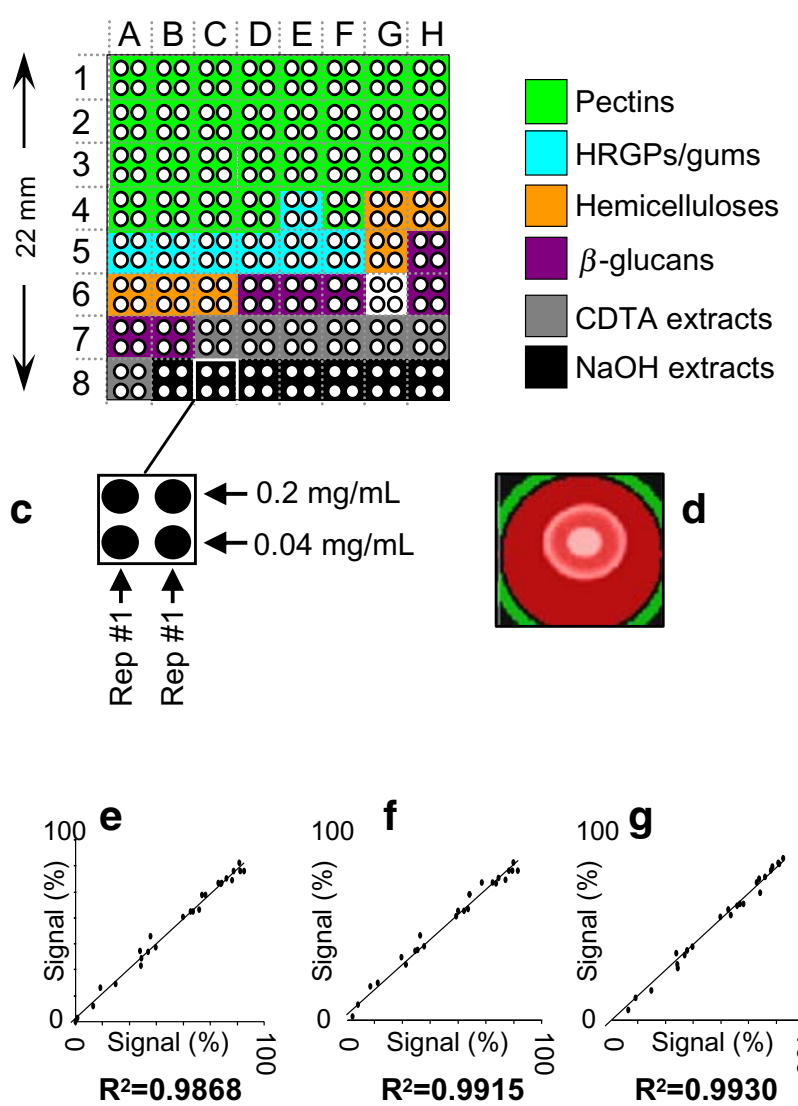

$100 \mathbf{9}$

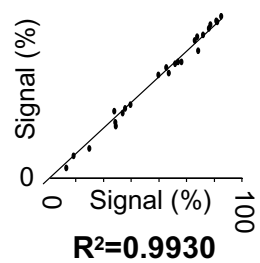

Fig. 1 Microarrays of plant cell wall glycans. a, b A library of 50 glycan polymers were spotted onto nitrocellulose membranes in an area $22 \mathrm{~mm} \times 22 \mathrm{~mm}$. Arrays included pectins, HRGPs, hemicelluloses and glucans (samples, A1-B7) and the position of these classes of polymer on the arrays are indicated by colour coding in (b). Fourteen samples extracted from Arabidopsis thaliana using either CDTA (samples $\mathrm{C} 7-\mathrm{A} 8$ ) or $\mathrm{NaOH}$ (samples $\mathrm{B} 8-\mathrm{H} 8$ ), were also included. Details of the samples are provided in Table 2. c Each sample was printed at the two concentrations indicated and in duplicate. d Microarray analysis software (ImaGene 6.0) was used to quantify spot signals and individual spot signals were defined as the mean pixel value within each spot area (red zone) minus the median pixel value in the surrounding local background area (green zone). $\mathbf{e}-\mathbf{g}$ Two pairs of three arrays were printed and probed with the anti-homogalacturonan mAb JIM5 on three separate occasions. The mean sample values (MSVs) for each pair were quantified and plotted against MSVs of an array produced on a different occasion. These are presented as scatter plots, with the $R^{2}$-values for the plots indicated

mapper software (http://bbc.botany.utoronto.ca/ntools/cgi-bin/ ntools heatmapper.cgi). For each antibody in the heatmap, the maximal TMSV was set to $100 \%$ and all other values within that data set adjusted accordingly. A cut off of $5 \%$ of the maximal TMSV was imposed. Hierarchical cluster analysis was performed on TMSVs obtained for 23 previously characterized antibodies and 7 newly produced ones using Epclust software (http://ep.ebi.ac.uk/EP/EPCLUST/). Clustering was based on correlation measure based distance and average linkage. 
Indirect immunofluorescence labeling of plant materials

Resin embedded and fresh plant material was sectioned and labelled with antibodies as described previously [26, 27]. LM14 and LM13 hybridoma supernatants were used as 1/ 10 dilutions in 5\%MPBS. Sections were counterstained for cellulose with Calcofluor white (fluorescent brightener 28, Sigma, Poole, UK) used as a $0.005 \%$ aqueous solution.

\section{Competitive-inhibition ELISA assays}

The ability of arabinose, galactose, rhamnose and oligoarabinosides to inhibit the binding of LM14 and LM13 to the immuogen in ELISAs was assessed as described previously [28].

Sugar composition analysis of modified pectic hairy regions (MHRs)

Sugar composition was determined using methanolysis as described previously [29]. MHRs were treated with $2 \mathrm{~N}$ $\mathrm{HCl}$ in dry methanol for $16 \mathrm{~h}$ at $80^{\circ} \mathrm{C}$, followed by $1 \mathrm{~h}$ of $2 \mathrm{M} \mathrm{CF}_{3} \mathrm{CO}_{2} \mathrm{H}$ (TFA) at $121^{\circ} \mathrm{C}$. The released sugars were analysed using high-performance anion exchange chromatography (HPAEC) with pulsed amperometric detection (PAD) as described previously [30].
Fig. 2 Probing of cell wall glycan arrays with $\mathrm{mAbs}$ with known specificities. a Glycan arrays were probed with 23 mAbs with previously defined specificities and 5 representative examples are shown: PAM1 (anti-homogalacturonan); BS400-2 (anti- $(1 \rightarrow 3)-\beta$-glucan; LM5 (anti-( $1 \rightarrow 4)-\beta$-galactan); JIM13 (anti-arabinogalactanprotein); JIM5 (anti-homogalacturonan). A control array was probed with secondary antibody, but no primary mAb. Arrays were scanned and converted into 16 bit greyscale TIFFs. b Heatmap showing the total mean sample values (TMSVs) for the binding of each mAb to each sample. The maximal TMSV for each mAb was set to 100 and all other values were adjusted accordingly. A cut off of $5 \%$ of the maximal TMSV was imposed and all values at, or below this are represented as black boxes a
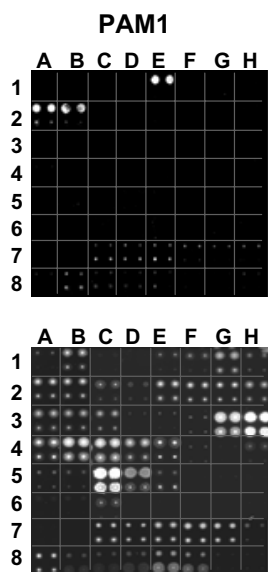

JIM13
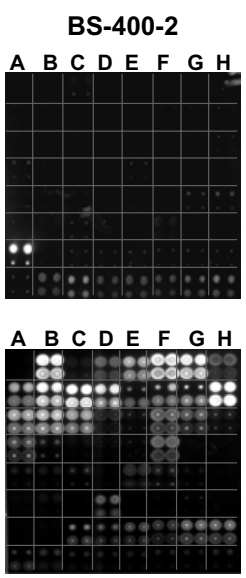

JIM5
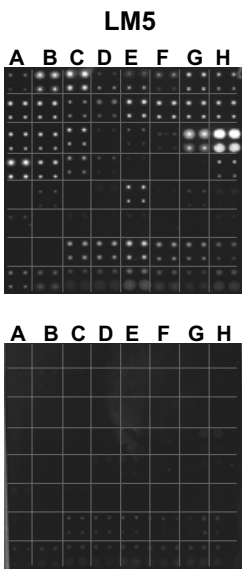

No mAb

b
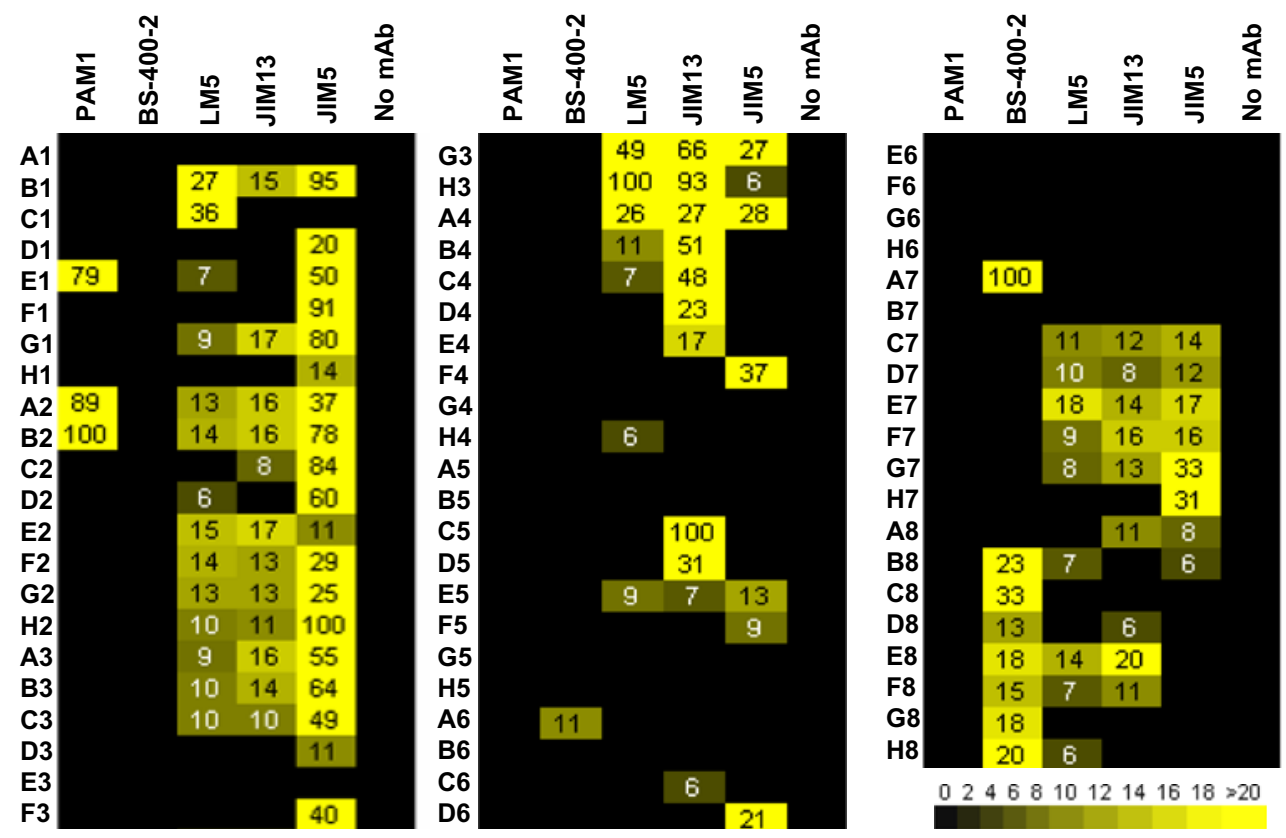


\section{Results}

Production of microarrays of plant cell wall polymers

The layout of the arrays is shown in Fig. 1 and colour coding indicates the distribution on the arrays of different classes of cell wall polymer (Fig. 1a,b). A detailed list of the samples used is provided in Table 2. Each sample was represented on the arrays by four spots (two concentrations, printed in duplicate) (Fig. 1c) and microarray analysis software was used to quantify background-corrected spot signals (Fig. 1d). As shown by the arrays in Figs. 2a and 3, the consistency of the printing and probing replicates within arrays was high. However, reproducibility between arrays was also tested. A total of six arrays $(3 \times 2$ pairs $)$ were printed and probed with the anti-HG mAb JIM5 on three separate occasions. The MSVs were quantified and the values for each array were plotted against those from an array produced on a different occasion. The $R^{2}$-values obtained for these plots were all $>0.98$, indicating a high degree of reproducibility between arrays (Fig. 1e-g).

\section{Probing of plant cell wall glycan arrays}

Arrays were probed with a range of mAbs with previously defined specificities for epitopes occurring on the major classes of cell wall polymers. Details of the mAbs used are provided in Table 1. A total of $23 \mathrm{mAbs}$ were tested and 5 representative examples are shown in Fig. 2a. The TMSVs for the binding of these mAbs to each sample (derived from three independent experiments) are shown as a heatmap in Fig. 2b, where spot signals are correlated to colour

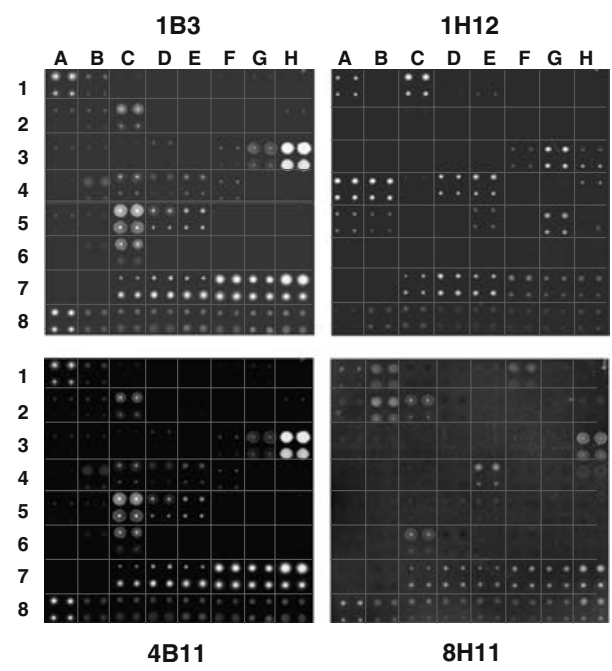

Fig. 3 Probing of cell wall glycan arrays with newly produced mAbs. Glycan arrays were probed with mono- or multiclonal antibodies generated following shotgun immunisation with plant cell wall polymers. Four representative examples are shown. Arrays were scanned and converted into 16 bit greyscale TIFFs
Fig. 4 Cluster analysis of antibody binding profiles to arrays. 23 mAbs with previously defined specificities, and 7 newly produced antibodies (red diamonds in black boxes) were analyzed using Epclust software. Hierarchical clustering was performed using clustered correlation measure based distance and average linkage. The binding profiles of all the mAbs analyzed clustered into four broad groups: 1 homogalacturonan-binders; 2 pectic side chain-binders; 3 HRGPbinders; and 4 hemicellulose-binders. Three mAbs were non-clustering outliers (NCO). The specificities of the 23 previously defined mAbs are listed in Table 1

intensity. mAb binding profiles were in good agreement with the published results for the antibodies tested. For example, mAb PAM1 has specificity for $\mathrm{HG}$ with a low degree of methyl-esterification (DE) [31] and consistent with this, PAM1 bound to lime pectin samples with DEs of 11-19\% (samples E1, A2 and B2). PAM1 did not bind above background to other pectin samples with higher DEs, or to any other cell wall glycans. mAb BS-400-2 is specific for $(1 \rightarrow 3)$ - $\beta$-glucan [32] and, of the defined samples, only bound to this polymer (sample A7) on the arrays. $(1 \rightarrow 3)$ $\beta$-glucan is known to be extractable from plant cell walls using $\mathrm{NaOH}$ and consistent with this, BS-400-2 also bound to weakly to $\mathrm{NaOH}$-solubilized extracts from $A$. thaliana (samples B8-H8). As expected, the epitopes recognized by some mAbs were detected on multiple samples and this was the case for mAbs LM5 (anti-( $1 \rightarrow 4)-\beta$-galactan), JIM13 (anti-AGP) and JIM5. However, the binding profiles of these mAbs were also consistent with their known specificities. For example, JIM5 bound with greatest avidity to pectin samples that contained abundant $\mathrm{HG}$ domains (such as the lime pectins E1-C3), but not to pectic fragments lacking $\mathrm{HG}$ (such as the arabinan or galactan samples $\mathrm{A} 1$ and $\mathrm{C} 1$ ).

To generate a new series of cell wall-directed mAbs, rats were immunized with an immunogen consisting of a crude extract of cell wall material solubilized from $A$. thaliana using CDTA. 13 cell lines were selected by an initial ELISA analysis against the immunogen and these were subsequently probed against the cell wall polymer arrays and four representative examples are shown in Fig. 3. Antibodies that bound with very low avidity, or appeared to have identical binding profiles to previously generated antibodies were not selected for further analysis whilst the data for the remaining seven were subjected to hierarchical cluster analysis.

\section{Hierarchical clustering of antibody binding profiles}

Cluster analysis was used to rapidly obtain information about the specificities of 7 new antibodies based on the similarly of their binding profiles on the arrays to 23 previously characterized mAbs (Fig. 4). Clustering indicated that most of the antibodies tested separated into 4 broad 
a

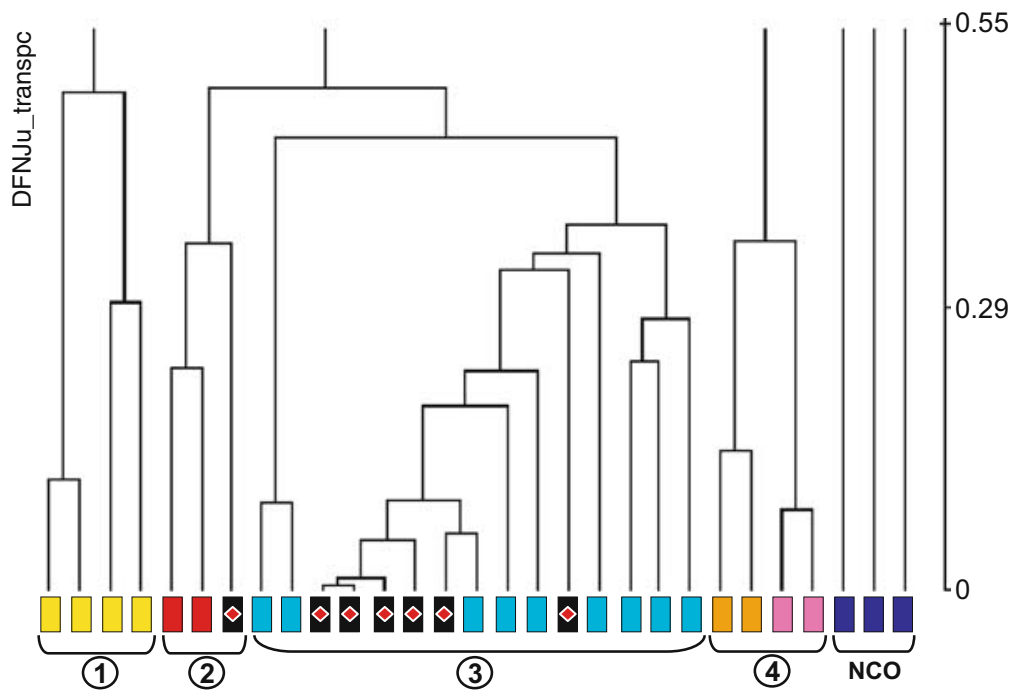

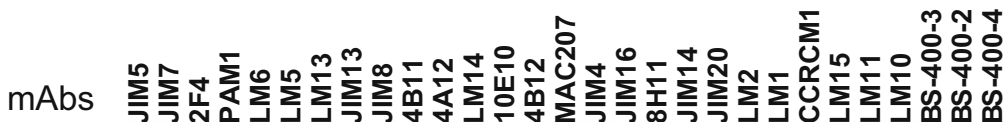

4
1
0
$\frac{0}{2}$
0
$\overline{0}$
$\overline{0}$
$\overline{3}$
$\overline{0}$
0
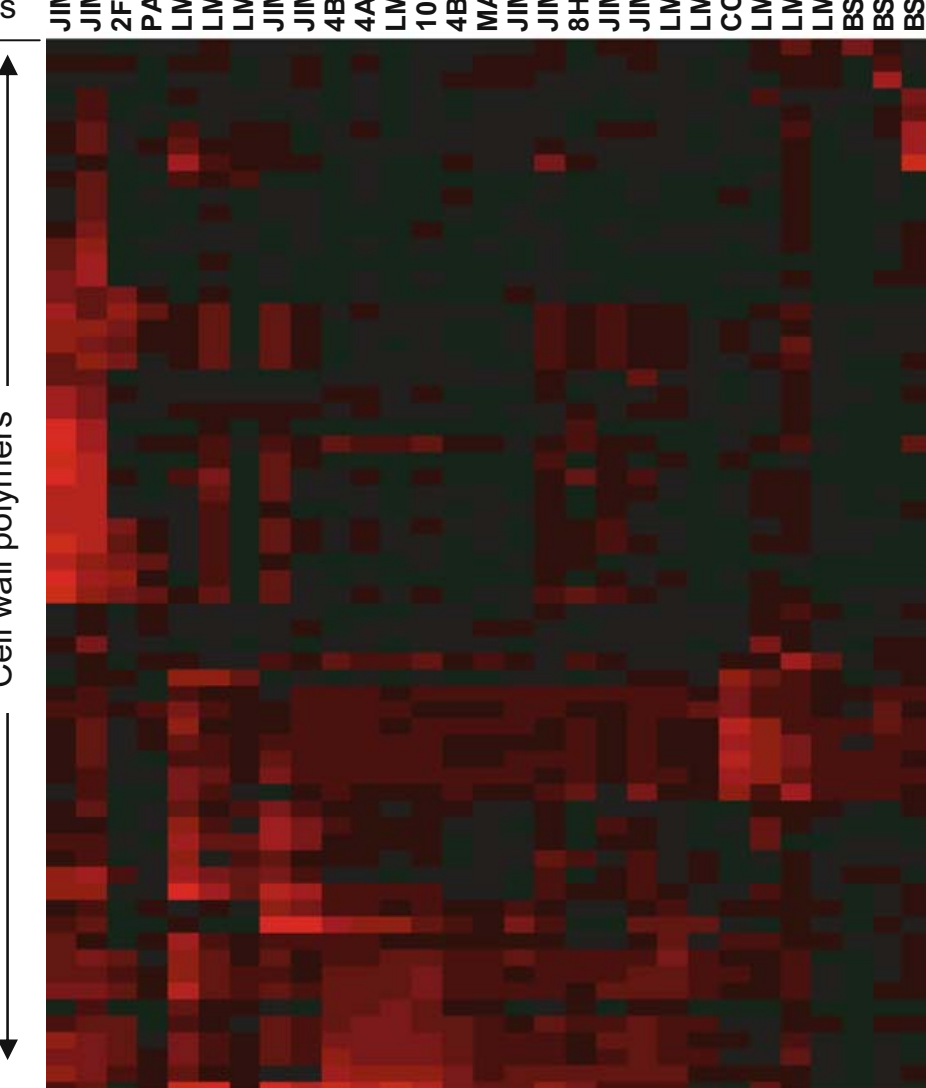

b

Pectins $\left\{\begin{array}{l}\square \text { Anti-homogalacturonan } \\ \square \text { Anti-pectic side chains }\end{array}\right.$

$\square$ Anti-HRGP

Hemicelluloses $\begin{cases}\square & \text { Anti-xyloglucan } \\ \square & \text { Anti-xylan/arabinoxylan }\end{cases}$

Anti-other cross linking polymers

$\Delta$ New antibodies 
clusters: HG-binders; pectic side chain-binders; HRGPbinders and hemicellulose-binders. Three antibodies with specificity for $(1 \rightarrow 3)(1 \rightarrow 4)-\beta$-glucan, $(1 \rightarrow 3)-\beta$-glucan and $(1 \rightarrow 4)-\beta$-mannan did not cluster with any other $m A b s$ within the 0.55 (distance measurement units) cut off imposed. These broad clusters were in agreement with the previously defined specificities of the mAbs tested, and this was also the case within sub-clusters. For example, within the HG-binders cluster, mAbs JIM5 and JIM7, which both bind to partially methyl-esterified HG [33], clustered closely, whilst mAbs 2F4 and PAM1, which both bind to non-methyl-esterified HG also clustered closely [31, 34]. Similarly, within the hemicellulose-binders, the anti-xylan mAbs LM10 and LM11 formed a sub-cluster, as did the two anti-xyloglucan mAbs CCRC-M1 and LM15. One of the new mAbs (1H12, designated LM13 when cloned) was grouped with the pectic side chain-binders LM5 (anti-( $1 \rightarrow 4)-\beta$-galactan) and LM6 (anti-( $(\rightarrow 5)$ - $\alpha$-arabinan). However, most of the new antibodies tested were grouped in the HRGP-binders cluster and within this, clustered most closely with the anti-AGP mAbs MAC207, JIM4 and JIM16. One of the new mAbs within this group (1B3, designated LM14 when cloned) was, together with LM13 subject to more detailed epitope characterization in order to further explore the evidence gained from the clustering analysis.

\section{Detailed characterization of the epitopes recognized} by LM14 and LM13

Cluster analysis of LM13 and LM14 suggested that the epitopes recognized by these mAbs may contain neutral sugars such as arabinose and galactose, that are abundant in the side chains of both pectins and HRGPs. The glycan arrays contained five 5 samples of branched (or 'hairy') regions of pectic polymers modified by enzymatic digestion ('modified hairy regions' or MHRs) that were rich in neutral sugars and for which monosaccharide composition was determined (Fig. 5). The binding of LM14 and LM13 to these samples was therefore of particular interest. LM14 bound only to an MHR from carrot (sample H3), whilst LM13 bound to MHRs from potato and apple (samples G3, A4 and B4). It was noteworthy that the two MHR samples to which LM13 did not bind (samples H3 and C4) had the lowest arabinose content, suggesting that the epitope recognized by this $\mathrm{mAb}$ may contain arabinose. In order to explore this possibility further, the sensitivity of the LM13 epitope to arabinanase digestion was investigated. To do this, cell wall glycan microarrays were digested with arabinanase prior to probing with $\mathrm{mAbs}$, and the TMSVs obtained with and without digestion were compared. These data are presented as scatter plots (Fig. 6a-d) in which signals from enzyme treated arrays were plotted against signals from untreated arrays. As a positive control,

\begin{tabular}{|c|c|c|c|c|c|}
\hline a & 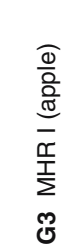 & 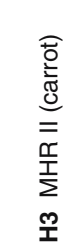 & 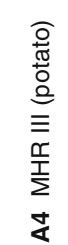 & 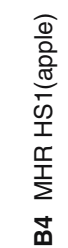 & 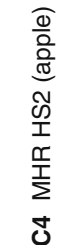 \\
\hline Fuc & 1 & 2 & 1 & nd & nd \\
\hline Rha & 14 & 20 & 13 & 9 & 11 \\
\hline Ara & 20 & 9 & 15 & 51 & 11 \\
\hline Gal & 12 & 24 & 23 & 10 & 20 \\
\hline Glc & 0 & 1 & 4 & 0 & 3 \\
\hline Man & nd & nd & nd & nd & nd \\
\hline Xyl & 13 & 1 & 1 & 8 & 18 \\
\hline GalA & 39 & 40 & 44 & 22 & 37 \\
\hline GlcA & 1 & 3 & 0 & nd & nd \\
\hline Rha:GalA & 0.35 & 0.51 & 0.29 & 0.41 & 0.30 \\
\hline Total (w/w\%) & 77 & 70 & 59 & 86 & 78 \\
\hline LM14 & & 100 & & & \\
\hline LM13 & 89 & & 100 & 97 & \\
\hline
\end{tabular}

Fig. 5 Binding of LM13 and LM14 to samples to pectic modified hairy regions (MHRs). a Monosaccharide composition of 5 pectin modified hairy regions (MHRs) from apple (samples G3, B4 and C4), carrot (sample H3), potato (sample A4). b TMSVs for the binding of mAbs LM14 and LM13 to these samples. The maximal TMSV for LM13 and LM14 (to all samples on the array) was set to 100 and all other values adjusted accordingly. A cut off of $5 \%$ of the maximal TMSV was imposed and all values at, or below this are represented as black boxes

arabinanase digested and untreated arrays were probed with the anti- $(1 \rightarrow 5)-\alpha$-arabinan mAb LM6 [24]. As expected, the binding of LM6 to all samples was reduced to some extent by arabinanase digestion (Fig. 6a). However, the effect of arabinanase digestion on LM13 binding was even more pronounced than for LM6 and LM13 binding to all samples on the arrays was essentially abolished (Fig. 6b). This suggested that the epitope recognized by LM13 either contained arabinan and was degraded by arabinanase, or that the epitope was attached to arabinan and was released from arrays by arabinanase. In contrast, LM14 binding to all samples was essentially unaffected by arabinanase digestion (Fig. 6c). The effect of galactanase digestion on $\mathrm{mAb}$ binding to arrayed samples was also assessed. LM13 binding was reduced to some extent by galactanase digestion, but the reduction was far less for than arabinanase digestion (Fig. 6d). LM14 binding to all samples was unaffected by galactanase digestion, and the ability of this enzyme to degrade galactan was indicated by the fact that the binding of the anti- $(1 \rightarrow 4)-\beta$-galactan mAb LM5 was essentially abolished by galactanase digestion (data not shown). The epitope recognized by LM13 was further characterized using competitive inhibition ELISA (ciELISA) assays (Fig. 6e). The binding of LM13 to immobilized arabinan was inhibited to $50 \%$ of maximal binding by approximately $450 \mu \mathrm{g} / \mathrm{ml}$ of both arabino- $\alpha 1,5$-octaose and arabino- $\alpha 1,5$-pentaose, but oligoarabinosides of lower 

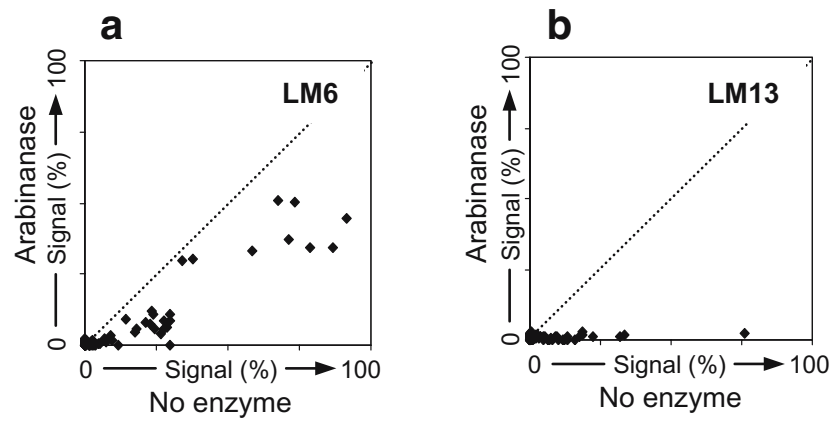

Cluster analysis suggested that LM13 was most similar to that of mAbs with specificity for pectic side chains, whilst the binding of LM14 was most similar to that of antiAGP mAbs. AGPs can be distinguished from pectic polymers using SDS-PAGE because whereas AGPs resolve as characteristic smears, pectic polymers typically do not enter gels [35]. Western blots were prepared using the same A. thaliana CDTA-extracted material that was used for immunisation and probed with LM13 or LM14 (Fig. 7). LM13 did not bind to material on these blots whereas LM14 bound to a high molecular weight smear $(\sim 70$ $200 \mathrm{kDa}$ ) that is characteristic of AGPs.
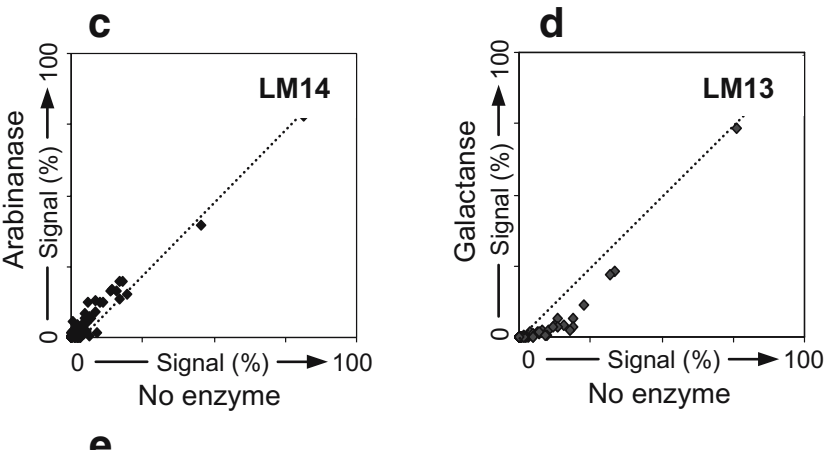

Immunolocalization of the LM14 and LM13 epitopes

In order to further investigate the epitopes recognized by LM13 and LM14 in planta a range of plant materials were probed with these mAbs using indirect immunofluorescence labelling. In transverse resin-embedded sections of $A$. thaliana inflorescence stems, LM13 bound specifically to epidermal cells and most strongly to the tangential cell walls (Fig. 8a). Plant cell wall glycans may be altered during extraction and/or immobilization such that epitopes are changed from their native state in planta. In order to test if this was a factor in determining the arabinase sensitivity of the LM13 epitope, sections of $A$. thaliana stems were also digested with arabinanase prior to probing with LM13. As shown by comparison of Fig. 8a,b, the binding of LM13 to A. thaliana stem epidermal cell walls was abolished following arabinanase digestion indicating that the epitopestructure immobilised on arrays recognized by LM13 reflected that observed in planta. In contrast to LM13, LM14 bound to some extent to all cell walls in an equivalent section through an $A$. thaliana stem (Fig. 8d). Labelling of the surface of intact roots of $A$. thaliana seedlings indicated that LM13 bound very weakly whereas LM14 bound strongly to the surface of root hairs (Fig. 8e and $\mathrm{f}$ ). In transverse sections through tobacco stems, the LM13 epitope was associated with cell walls adjacent to metaxylem cells of the vascular tissue-a distinctly different labelling pattern to that observed in $A$. thaliana stems (Fig. 8g). In contrast, LM14 did not label any cell walls in tobacco stems (Fig. 8h).

\section{Discussion}

The work presented here demonstrates the potential of glycan microarrays for overcoming a major bottleneck in anti-glycan $\mathrm{mAb}$ production. Specifically, the use of microarrays enabled $50 \mu \mathrm{l}$ of hybridoma supernatant to be screened rapidly and simultaneously against $>60$ potential

epitope-bearing target molecules. A novel aspect of this

degrees of polymerisation and rhamnose, galactose and glucuronic acid failed to inhibit binding at the highest concentration tested of $1 \mathrm{mg} / \mathrm{ml}$; . The binding of LM14 to immobilized antigen was not inhibited by any of the haptens tested at concentrations up to $1 \mathrm{mg} / \mathrm{ml}$ (data not shown). 
Fig. 7 Immunoblot of monoclonal antibodies LM13 and LM14. The CDTA-soluble Arabidopsis thaliana fraction that was used as the immunogen was separated by SDS-PAGE with $30 \mu \mathrm{g}$ protein loading per lane. A Western blot of this material was probed with LM13 and LM14. LM13 did not bind to the blot but LM14-probing produced a smear in the region of 70 $200 \mathrm{kDa}$, which is characteristic of AGPs

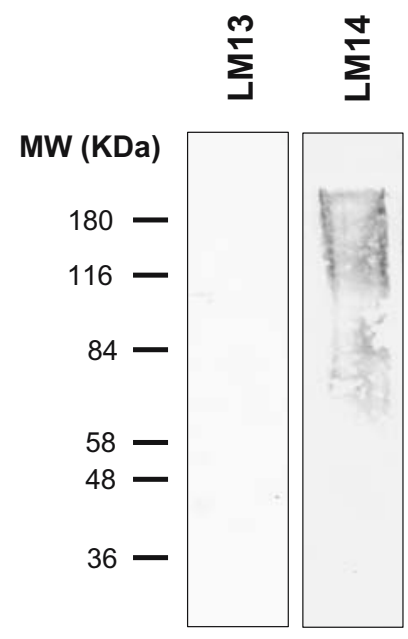

work was the use of cluster analysis of array data to rapidly predict antibody specificities by comparison with previously defined mAbs. Subsequent detailed analyses of the specificities of the new mAbs LM13 and LM14 indicated they bound to pectic and AGP class of polymers respectively, as was predicted by the cluster analysis. These results indicate that if a relatively large set of probes with defined specificities are available to serve as references, this is an effective method for high-throughput initial $\mathrm{mAb}$ screening. One potential limitation of shotgun immunisation could be immuno-dominance, such that an immune response is elicited against a limited subset of the injected antigens. In this study two mAbs, LM13 and LM14 were selected with specificity for two different classes of molecule. However, of the seven mAbs selected for cluster analysis of binding profiles, six clustered with $\mathrm{mAbs}$ with specificity for AGPs and one with mAbs to pectic side chains, suggesting that AGPs were the immuno-dominant antigens in this case. It is therefore likely that such a multiantigen approach towards cell wall polymers may be most effective to obtain a panel of mAbs with a range of specificities within a single class of polymer.

Fig. 8 Indirect immunofluorescence labelling of plant material with LM14 and LM13. LM13 bound strongly to inner and outer radial walls of epidermal cells in transverse sections of $A$. thaliana inflorescence stem sections. The section is counterstained with calcofluor (blue) that labels $\beta$-linked glucans. b LM13 binding to $A$. thaliana inflorescence stem sections when sections were treated with arabinanase. Arrowheads in $\mathbf{a}$ and $\mathbf{b}$ indicate the epidermal surface. c Control in which an equivalent stem to that in a was labelled with the absence of primary antibody. d, An equivalent section to that shown in a labelled with LM14 which bound to all cell walls. e LM13 bound very weakly to the intact surface of the roots of $A$. thaliana seedlings. f LM14 bound strongly to the surface of root hairs (arrowheads) of Arabidopsis seedling roots. $\mathrm{g}$ In transverse sections through tobacco stem LM13 bound strongly to cell walls adjacent to files of metaxylem cells $(m x)$. h LM14 did not bind to equivalent sections through tobacco stems. Scale bars $=50 \mu \mathrm{m}$ for $\mathbf{a}-\mathbf{d}, \mathbf{g}$ and $\mathbf{h} ; 20 \mu \mathrm{m}$ for $\mathbf{g}$ inset, $50 \mu \mathrm{m}$ for $\mathbf{e}$ and $\mathbf{f}$
The arrays we constructed were based on the noncovalent attachment of glycans to nitrocellulose. This approach has the advantage that molecules can be immobilized directly without the need to create functional groups, and nitrocellulose has been previously shown to be a effective substrate for the immobilization of diverse glyans $[35,36]$. Non-covalent attachment has the potential drawback that variations in the effectiveness of immobilization may result from differences in the structural
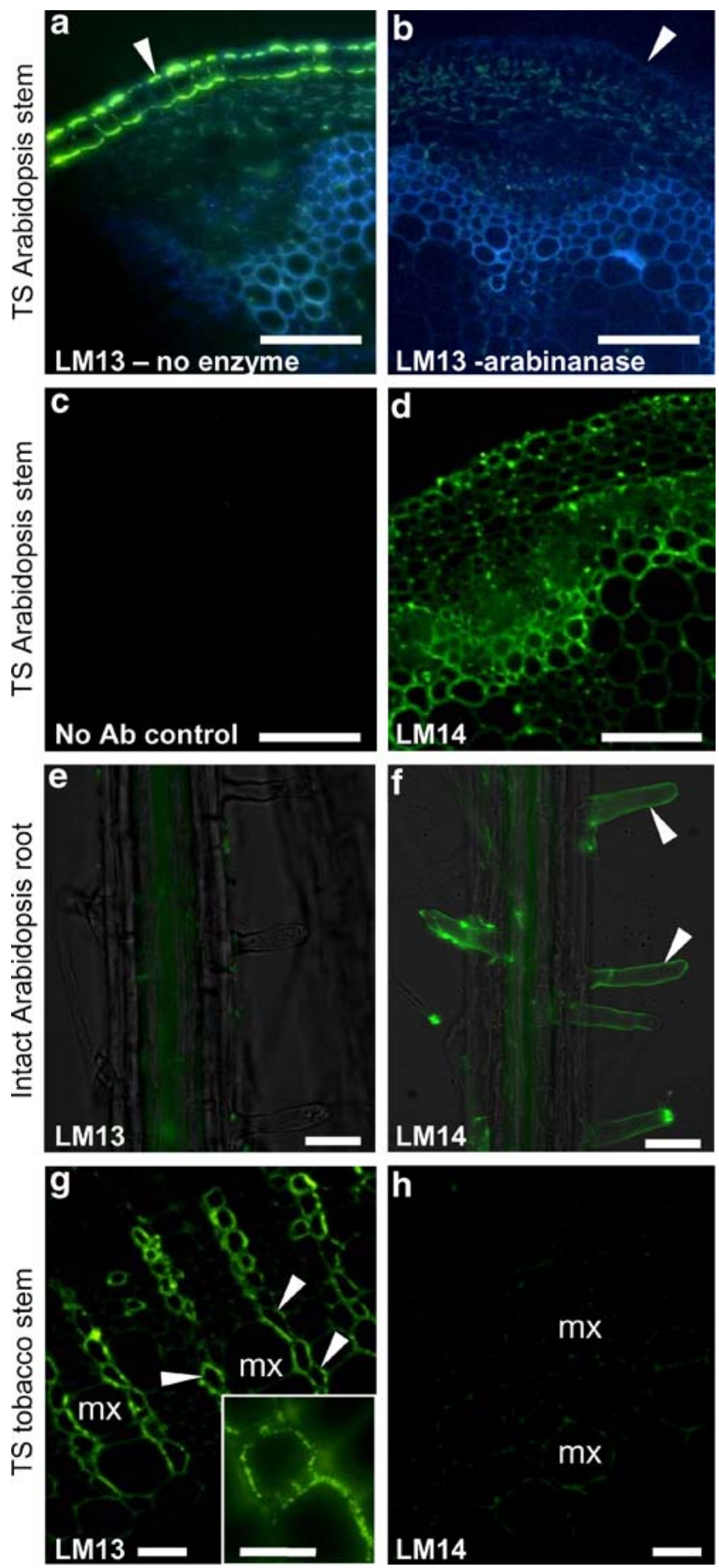
properties of the arrayed molecules [8]. All of the arrayed samples used in this work were recognized to some extent by at least one of the mAbs used (as shown in Fig. 4), indicating that all were immobilized to some degree. Nevertheless, the spot signals obtained can only provide semi-quantitative information about $\mathrm{mAb}$ binding.

Two new probes, LM13 and LM14 were produced against cell wall polymers and both have distinctly different specificities to previously generated antibodies. Cluster analysis, ciELISA data immunoblotting and arabinanase sensitivity suggested that the epitope recognized by LM13 is an arabinan-containing structure that occurs as a side chain on pectic polymers. Pectic side chains typically consist of either galactan, arabinan or type I arabinogalactan chains in which arabinose usually occurs as a terminal sugar [18]. It was of note that LM13 binding to arrays was reduced to some extent by galactanase digestion. However, the galactanase used had very low arabinanase side activity $(<0.03 \mathrm{U} / \mathrm{ml}$ specific activity with an arabinan substrate compared to $780 \mathrm{U} / \mathrm{ml}$ specific activity with a galactan substrate). It is possible therefore that galactanase digestion resulted in the indirect loss of the LM13 epitope by cleavage of galactan to which an arabinan-containing epitope is attached. The fact that LM14 bound to both pectin-derived MHR samples and to AGP-like material on a blot suggests that this $\mathrm{mAb}$ binds to an epitope of type II arabinogalactan that may occur on both pectins and AGPs. The novel patterns of recognition on A. thaliana and other plant materials indicates that these are useful new probes for the analysis of cell wall glycans polymers and complex cell wall architectures.

Acknowledgments Thanks to Mike Hahn for mAb CCRC-M1 and samples G4 and F4 and to Marie-Christine.Ralet and Jean-François Thibault for samples D1 and F3.

\section{References}

1. Willats, W.G., Rasmussen, S.E., Kristensen, T., Mikkelsen, J.D. Knox, J.P.: Sugar-coated microarrays: A novel slide surface for the high-throughput analysis of glycan. Proteomics 2, 1666-1671 (2002)

2. Feizi, T., Chai, W.: Oligosaccharide microarrays to decipher the glyco code. Nat. Rev. Mol. Cell Biol. 5, 582-588 (2004)

3. Wang, D., Liu, S., Trummer, B.J., Deng, C., Wang, A.: Carbohydrate microarrays for the recognition of cross-reactive molecular markers of microbes and host cells. Nat. Biotechnol. 20, 275-281 (2002)

4. Feizi, T., Mulloy, B.: Carbohydrates and glycoconjugates. Glycomics: The new era of carbohydrate biology. Curr. Opin. Struct. Biol. 13, 602-604 (2003)

5. Wang, D.: Carbohydrate microarrays. Proteomics 3(11), 2167$2175(2003)$

6. Feizi, T., Fazio, F., Chai, W., Wong, C.H.: Carbohydrate microarrays - a new set of technologies at the frontiers of glycomics. Curr. Opin. Struct. Biol. 13(5), 637-645 (2003)

7. Hirabayashi, J.: Oligosaccharide microarrays for glycomics. Trends Biotechnol. 21(4), 141-143 (2003)
8. Larsen, K., Thygesen, M.B., Guillaumie, F., Willats, W.G., Jensen, K.J.: Solid-phase chemical tools for glycobiology. Carbohydr. Res. 341(10), 1209-1234 (2006)

9. Ratner, D.M., Adams, E.W., Disney, M.D., Seeberger, P.H.: Tools for glycomics: Mapping interactions of carbohydrates in biological systems. ChemBioChem 5(10), 1375-1383 (2004)

10. Kohler, G., Milstein, C.: Continuous cultures of fused cells secreting antibody of predefined specificity. Nature 256, 495-497 (1975)

11. Paramithiotis, E., Pinard, M., Lawton, T., LaBoissiere, S., Leathers, V.L., Wen-Quan Zou, W.-Q., Estey, L.A., Lamontagne, J., Lehto, M.T., Kondejewski, L.H., Francoeur, G.P., Papadopoulos, M., Haghighat, A., Spatz, S.J., Head, M., Will, R., Ironside, J., O'Rourke, K., Tonelli, Q., Ledebur, H.C., Chakrabartty, A., Cashman, N.R.: A prion protein epitope selective for the pathologically misfolded conformation. Nat. Med. 9, 893-899 (2003)

12. Carpita, N.C., Gibeaut, D.M.: Structural models of primary cell walls in flowering plants: Consistency of molecular structure with the physical properties of the walls during growth. Plant J. 3, 1-30 (1993)

13. Ridley, B.L., O’Neill, M.A., Mohnen, D.: Pectins: Structure, biosynthesis, and oligogalacturonide-related signaling. Phytochemistry 57, 929-967 (2001)

14. Chapple, C., Carpita, N.: Plant cell walls as targets for biotechnology. Curr. Opin. Plant Biol. 1, 179-185 (1998)

15. Farrell, A.E., Plevin, R.J., Turner, B.T., Jones, A.D., O'Hare, M., Kammen, D.M.: Ethanol can contribute to energy and environmental goals. Science 311, 506-508 (2006)

16. Willats, W.G.T., Knox, J.P., Mikkelsen, J.D.: Pectin: New insights into an old polymer are starting to gel. Trends Food Sci. Technol. 17(3), 97-104 (2006)

17. Fry, S.C.: Primary cell wall metabolism: Tracking the careers of wall polymers in living plant cells. New Phytol. 161, 641-675 (2004)

18. Willats, W.G., McCartney, L., Mackie, W., Knox, J.P.: Pectin: Cell biology and prospects for functional analysis. Plant Mol. Biol. 47 (1-2), 9-27 (2001)

19. Showalter, A.M.: Arabinogalactan-proteins: Structure, expression and function. Cell. Mol. Life Sci. 58(10), 1399-1417 (2001)

20. Knox, J.P.: The extracellular matrix in higher plants. 4. Developmentally regulated proteoglycans and glycoproteins of the plant cell surface. FASEB J. 9(11), 1004-1012 (1995)

21. Kieliszewski, M.J., Lamport, D.T.: Extensin: Repetitive motifs, functional sites, post-translational codes, and phylogeny. Plant J. 5 (2), 157-172 (1994)

22. Knox, J.P.: The use of antibodies to study the architecture and developmental regulation of plant cell walls. Int. Rev. Cytol. 171, 79-120 (1997)

23. Fry, S.: The Growing Plant Cell Wall: Chemical and Metabolic Analysis, p. 64. Blackburn Press, New Jersey (2000)

24. Willats, W.G.T., Marcus, S.E., Knox, J.P.: Generation of a monoclonal antibody specific to (1-5)-a-L-arabinan. Carbohydr. Res. 308, 149-152 (1998)

25. Bazin, H.: Production of rat monoclonal antibodies with the LOU rat non-secreting IR883F myeloma cell line. Prot. Biol. Fluids 29, 615-618 (1982)

26. Willats, W.G., Orfila, C., Limberg, G., Buchholt, H.C., van Alebeek, G.J., Voragen, A.G., Marcus, S.E., Christensen, T.M., Mikkelsen, J.D., Murray, B.S., Knox, J.P.: Modulation of the degree and pattern of methyl-esterification of pectic homogalacturonan in plant cell walls. Implications for pectin methyl esterase action, matrix properties, and cell adhesion. J. Biol. Chem. 276 (22), 19404-19413 (2001)

27. Willats, W.G., McCartney, L., Steele-King, C.G., Marcus, S.E., Mort, A., Huisman, M., van Alebeek, G.J., Schols, H.A., Voragen, A.G., Le Goff, A., Bonnin, E., Thibault, J.F., Knox, J.P.: A xylogalacturonan epitope is specifically associated with plant cell detachment. Planta 218, 673-681 (2003) 
28. Willats, W.G., Limberg, G., Buchholt, H.C., van Alebeek, G.J., Benen, J., Christensen, T.M., Visser, J., Voragen, A., Mikkelsen, J.D., Knox, J.P.: Analysis of pectic epitopes recognised by hybridoma and phage display monoclonal antibodies using defined oligosaccharides, polysaccharides, and enzymatic degradation. Carbohydr. Res. 327 (3), 309-320 (2000)

29. De Ruiter, G.A., Schols, H.A., Voragen, A.G.J., Rombouts, F.M.: Anal. Biochem. 207, 176-185 (1992)

30. Verhoef, R., de Waard, P., Schols, H.A., Rättö, M., Siika-aho, M., Voragen, A.G.J.: Carbohydr. Res. 337(20), 1821-1831 (2002)

31. Willats, W.G., Gilmartin, P.M., Mikkelsen, J.D., Knox, J.P.: Cell wall antibodies without immunization: Generation and use of de-esterified homogalacturonan block-specific antibodies from a naive phage display library. Plant J. 18, 57-65 (1999)

32. Meikle, P.J., Bonig, I., Hoogenraad, N.J., Clarke, A.E., Stone, B. A.: The location of (13)- $\beta$-glucans in the walls of pollen tubes of Nicotiana alata using a (13)- $\beta$-glucan-specific monoclonal antibody. Planta 185, 1-8 (1991)

33. Clausen, M.H., Willats, W.G.T., Knox, J.P.: Synthetic methyl hexagalacturonate hapten inhibitors of anti-homogalacturonan monoclonal antibodies LM7, JIM5 and JIM7. Carbohydr. Res. 338, 1797-1800 (2003)

34. Liners, F., Letesson, J.J., Didembourg, C., Van Cutsem, P.: Monoclonal antibodies against pectin: Recognition of a conformation induced by calcium. Plant Physiol. 91, 1419-1424 (1989)

35. Orfila, C., Huisman, M.M., Willats, W.G., van Alebeek, G.J., Schols, H.A., Seymour, G.B., Knox, J.P.: Altered cell wall disassembly during ripening of $\mathrm{Cnr}$ tomato fruit: Implications for cell adhesion and fruit softening. Planta 215, 440-447 (2002)

36. Wang, D., Liu, S., Trummer, B.J., Deng, C., Wang, A.: Carbohydrate microarrays for the recognition of cross-reactive molecular markers of microbes and host cells. Nat. Biotechnol. 20, 275-281 (2002)

37. Jones, L., Seymour, G.B., Knox, J.P.: Localization of pectic galactan in tomato cell walls using a monoclonal antibody specific to (1-4)-[beta]-D-galactan. Plant Physiol. 113, 1405-1412 (1997)
38. Puhlmann, J., Bucheli, E., Swain, M.J., Dunning, N., Albersheim, P., Darvill, A.G., Hahn, M.G.: Generation of monoclonal antibodies against plant cell-wall polysaccharides. I. Characterization of a monoclonal antibody to a terminal alpha- $(1 \rightarrow 2)$-linked fucosyl-containing epitope. Plant Physiol. 104, 699-710 (1994)

39. Pettolino, F.A., Hoogenraad, N.J., Ferguson, C., Bacic, A., Johnson, E., Stone, B.A.: A (1-4)-beta-mannan-specific monoclonal antibody and its use in the immunocytochemical location of galactomannans. Planta 214, 235-242 (2001)

40. McCartney, L., Marcus, S.E., Knox, J.P.: Monoclonal antibodies to plant cell wall xylans and arabinoxylan. J. Histochem. Cytochem. 53, 543-546 (2005)

41. Meikle, P.J., Bonig, I., Hoogenraad, N.J., Clarke, A.E., Stone, B.A.: A (1-3,1-4)-beta-glucan-specific monoclonal antibody and its use in the quantitation and immunocytochemical location of (13,1-4)-beta-glucans. Planta 188, 1-8 (1991)

42. Smallwood, M., Yates, E.A., Willats, W.G.T., Martin, H., Knox, J.P.: Immunochemical comparison of membrane-associated and secreted arabinogalactan-proteins in rice and carrot. Planta 198, 452-459 (1996)

43. Yates, E.A., Valdor, J.-F., Haslam, S.M., Morris, H.R., Dell, A., Mackie, W., Knox, J.P.: Characterization of carbohydrate structural features recognized by anti-arabinogalactan-protein monoclonal antibodies. Glycobiology 6, 131-139 (1996)

44. McCabe, P.F., Valentine, T.A., Forsberg, L.S., Pennell, R.I.: Soluble signals from cells identified at the cell wall establish a developmental pathway in carrot. Plant Cell 9, 2225-2241 (1997)

45. Smallwood, M., Martin, H., Knox, J.P.: An epitope of rice threonine- and hydroxyproline-rich glycoprotein is common to cell wall and hydrophobic plasma membrane glycoproteins. Planta 196, 510-522 (1995)

46. Smallwood, M., Beven, A., Donovan, N., Neill, S.J., Peart, J., Roberts, K., Knox, J.P.: Localization of cell wall proteins in relation to the developmental anatomy of the carrot root apex. Plant J. 5, 237-246 (1994) 